\title{
Chaperoning mitochondrial permeability transition: regulation of transition pore complex by a J-protein, DnaJC15
}

\author{
D Sinha ${ }^{1}$ and P D'Silva ${ }^{*}, 1$
}

Mitochondria have a central role in the intrinsic pathway of apoptosis and involve activation of several transmembrane channels leading to release of death factors. Reduced expression of a mitochondrial J-protein DnaJC15 was associated with the development of chemoresistance in ovarian cancer cells. DnaJC15 was found to be a part of mitochondrial protein-transport machinery, though its connection with cell death mechanisms is still unclear. In the present study, we have provided evidence towards a novel function of DnaJC15 in regulation of mitochondrial permeability transition pore (MPTP) complex in normal and cancer cells. Overexpression of DnaJC15 resulted in MPTP opening and induction of apoptosis, whereas reduced amount of protein suppressed MPTP activation, upon cisplatin treatment. DnaJC15 was found to exert its proapoptotic function through the essential component of MPTP, cyclophilin D (CypD). Our results reveal a specific role of DnaJC15 in recruitment and coupling of CypD with mitochondrial permeability transition. In summary, our analysis provides first-time insights on the functional connection between mitochondrial inner membrane protein translocation machinery-associated J-protein DnaJC15 and regulation of cell death pathways.

Cell Death and Disease (2014) 5, e1101; doi:10.1038/cddis.2014.72; published online 6 March 2014

Subject Category: Cancer

J-proteins serve as auxiliary factors in regulation of Hsp70 class of chaperones ${ }^{1}$ and have a central role in translocation of precursor proteins into organelles, such as mitochondria. ${ }^{2,3}$ Human mitochondria consist of two J-proteins DnaJC19 (JC19) and DnaJC15 (JC15) governing the primary protein import activity. ${ }^{4,5} \mathrm{JC} 19$ is ubiquitously expressed in all tissues, but the expression profile of $\mathrm{JC} 15$ is regulated by the methylation status of the $\mathrm{CpG}$ islands at the promoter region. ${ }^{6}$ Incidentally, JC15 was identified as a protein whose loss of expression was associated with development of chemoresistance in clinical samples of ovarian cancer., 7 Ovarian and breast cancer patients exposed to sustained chemotherapy showed lower levels of the protein and enhanced resistance to the drugs, which was attributed to methylation of the CpG islands of the gene. ${ }^{6,7}$ However, the role of mitochondrial JC15 in modulation of apoptotic pathways is still enigmatic.

Mitochondria have a key role in activation of apoptosis in mammalian cells, ${ }^{9}$ and dissipation of mitochondrial membranes leads to release of various proapoptotic molecules. ${ }^{10}$ The compartmentalization of these proteins into the organelle to spatially separate them from their interacting partners or targets acts as a safeguard mechanism. A specific death signal causing activation of transporter channels leading to loss of the membranous partitioning promote the release of the mitochondrial death inducers into the cytosol. ${ }^{11-15}$ One of the major transporters that get activated upon apoptotic stimuli is the mitochondrial permeability transition (MTP) pore (MPTP) complex.

MPTP has a key role in cell death by opening up the inner mitochondrial membrane through the formation of a nonspecific pore. Under physiological conditions, mitochondria maintain a robust transmembrane potential and low conductance state of MPTP channel. ${ }^{10} \mathrm{~A}$ prototypical MPTP is composed of voltage-dependent anion channel (VDAC), adenine nucleotide translocator (ANT) and a peptidyl-proline isomerase cyclophilin D (CypD). Recently, it has been proposed that MPTP complex is constituted by a dimer of $\mathrm{F}_{\mathrm{o}}-\mathrm{F}_{1}$ adenosine triphosphate (ATP) synthase, which is incorporated in the lipid bilayers to form $\mathrm{Ca}^{2+}$-activated channels. ${ }^{16}$ The regulator of MPTP, CypD associates with the lateral stalk of ATP synthase and binds with the OSCP (oligomycin sensitivity-conferring protein) subunit in a $\mathrm{Pi}$ (inorganic phosphate)-dependent manner. ${ }^{16}$ In response to pro-apoptotic stimuli, MPTP assumes a higher conductance state that allows disregulated entry of small molecules causing loss of potential, osmotic imbalance and organellar disintegration. ${ }^{12}$ Recent reports have shown VDAC and ANT to be dispensable for MPTP activation and mouse knocked out for either of the proteins still undergo permeability transition. ${ }^{17}$ Mouse lacking CypD show enhanced resistance to cell death and activation of the MPTP, highlighting its essential role at the channel. ${ }^{18}$ However, the exact molecular details of the process are still not identified.

\footnotetext{
${ }^{1}$ Department of Biochemistry, Indian Institute of Science, Bangalore, India

${ }^{*}$ Corresponding author: P D'Silva, Department of Biochemistry, Indian Institute of Science, C. V. Raman Avenue, Bangalore, Karnataka 560012, India. Tel: +91 080 22932821; Fax: +91 080 23600814; E-mail: patrick@ biochem.iisc.ernet.in

Keywords: mitochondria; J-proteins; permeability transition

Abbreviations: CypD, cyclophilin D; mtHsp70, mitochondrial heat-shock protein 70; MPTP, mitochondrial permeability transition pore; VDAC, voltage-dependent anion channel; ANT, adenine nucleotide translocator; ATP, adenosine triphosphate; CsA, cyclosporine A

Received 18.11.13; revised 22.1.14; accepted 27.1.14; Edited by M Agostini
} 
We found that mitochondrial JC15 other than its primary import function, has a critical role in modulation of cellular sensitivity to chemotherapeutic agents. JC15 modulates the activity of the MPTP complex by differentially recruiting CypD and its matrix interacting partner TRAP1 to the MPTP channel upon xenobiotic insult and induces apoptosis by channel opening. Additionally, we have also identified the domain of JC15 mediating its pro-apoptotic function. In summary, our results identify an additional function of mitochondrial membrane J-protein $\mathrm{JC} 15$ in regulation of the intrinsic pathways of apoptosis upon chemotherapeutic treatment of cancer cells.

\section{Results}

Variable expression of JC15 results in differential cellular sensitivity to cisplatin. Loss of JC15 expression has been previously implicated in the development of chemoresistance in ovarian carcinoma. ${ }^{6-8}$ Indeed, prolonged exposure of ovarian cancer-derived OVCAR-3 and breast cancer MCF7 cells to cisplatin led to progressive reduction of JC15 expression together with an increase in cell viability (Supplementary Figures S1A-D). However, loss of the protein expression was not limited to cisplatin alone. In support to previous observations on clinical samples, ${ }^{8,19}$ exposure of both OVCAR-3 and MCF7 cells to doxorubicin had a similar effect on the JC15 protein levels and led to development of chemoresistance in these cells
(Supplementary Figures S1E-H). Therefore, to adjudge the role of JC15 in cell death, we altered JC15 expression in HEK293T and assayed for sensitivity to the drug. We observed that loss of JC15 expression in the cells resulted in increased chemoresistance (Figure 1a). The cells showed suppression of apoptosis as suggested by lower annexinVPI-stained population and activity of effecter caspases 3 and 7 (Figures 1b and d). JC15-depleted cells also showed better maintenance of mitochondrial membrane potential under treated conditions as compared with controls (Figure 1c). This shows that depletion of JC15 protects the cells from cisplatin-mediated apoptotic cell death.

In contrast, overexpression of the protein (Supplementary Figure S2A) promoted cellular sensitivity to the drug and induced apoptosis as indicated by enhanced accumulation of annexinV-PI-stained cell population and increased caspase $3 / 7$ activity (Figures 1a, b and d). Consistently, JC15overexpressing cells showed increased amounts of cytosolic cytochrome $c$ and complete depolarization of mitochondrial inner membrane potential (Supplementary Figure S6A and Figure 1c). To extend our findings in cancer cells, a similar observation was found in OVCAR-3 and MCF7 cells presenting elevated amounts of $\mathrm{JC} 15$, in terms of cell viability (Supplementary Figures S3A-D and S4E-H) and maintenance of membrane potential (Supplementary Figure S5A-B).

To further validate the involvement of JC15 in the chemosensitive phenotype and to identify the domain
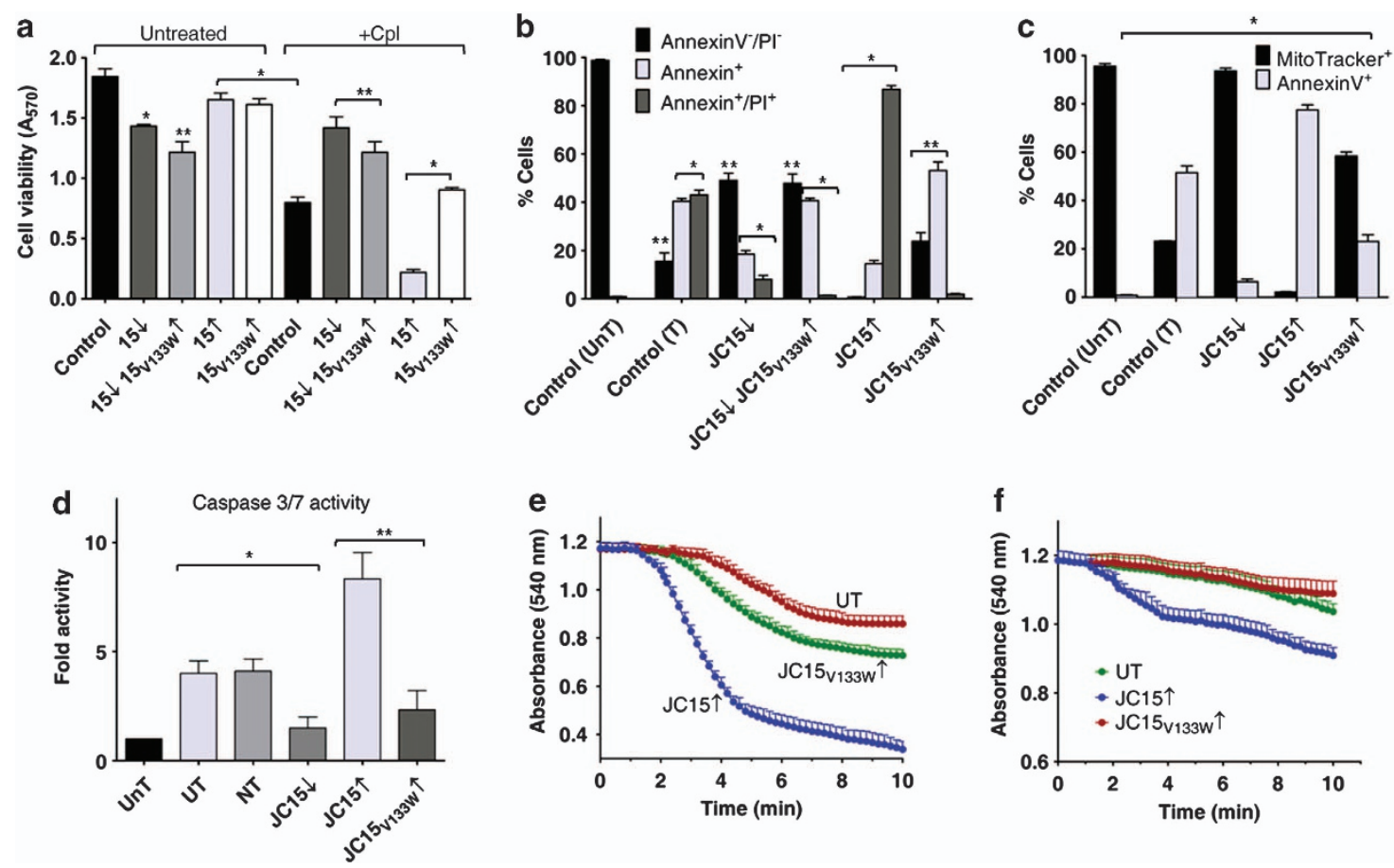

Figure 1 Overexpression of $\mathrm{JC} 15$ leads to enhanced chemosensitivity. (a) Relative viability of cells variably expressing JC15 or JC15 $5_{\mathrm{V} 133 \mathrm{~W}}$ were either left untreated or treated with cisplatin $(+\mathrm{Cpl})$ and measured using MTT assay. Data are represented as mean \pm S.E.M. $n=8,{ }^{*} P$ (two tailed) $<0.0001,{ }^{* *} P$ (two tailed) $<0.001$. (b and $\mathbf{c}$ ) Cisplatin-treated cells with altered levels of $\mathrm{JC} 15$ or $\mathrm{JC} 5_{\mathrm{V}_{133 \mathrm{w}}}$ mutant were dual stained with AnnexinV-AlexaFlour 488/propidium iodide (PI) (b) or AnnexinV-AlexaFlour 488/MitoTracker Red CMXRos (c). Bars represent mean \pm S.E.M. $n=3,{ }^{*} P$ (two tailed) $<0.0001$, ${ }^{* *} P$ (two tailed) $<0.001$; UnT, untreated untransfected cells; $\mathrm{T}$, untransfected cells treated with cisplatin. (d) Fold caspase $3 / 7$ activity of cells with altered wild-type or mutant JC15 expression over untransfected cells represented as mean \pm S.E.M. $n=3,{ }^{*} P$ (two tailed) $<0.0001,{ }^{* *} P$ (two tailed) $<0.001$; NT, non-targeting siRNA control; UT, cisplatin-treated untransfected cells. (e and f) Isolated mitochondria overexpressing JC15 and $\mathrm{JC}_{15} \mathrm{~V}_{\mathrm{V} 133 \mathrm{~W}}$ were induced to swell in the absence (e) or presence (f) of $1 \mathrm{nM}$ cyclosporin (CsA) and represented as mean \pm S.E.M. $n=3, P<0.0001$ with reference to UT 
responsible for the function, we overexpressed the loss of function $\mathrm{V} 133 \mathrm{~W}$ mutant of $\mathrm{JC} 15$ with a defective $\mathrm{J}$-domain (Supplementary Figure S2A). Overexpression of JC15 $\mathrm{V} 133 \mathrm{~W}$ exerted a partial dominant-negative effect on cell viability and induction of apoptosis (Figures $1 \mathrm{a}, \mathrm{b}$ and $\mathrm{d}$ ). The cells showed a fractional decrease in mitochondrial potential loss (Figure 1c) and subsequent cytochrome $c$ release (Supplementary Figure S6A). This highlights that the $\mathrm{J}$-domain of $\mathrm{JC} 15$ has an essential role in the development of chemosensitive phenotype.

JC15 modulates the activity of MPTP complex. As overexpression of $\mathrm{JC} 15$ is associated with complete dissipation of mitochondrial membrane potential and loss of the protein protects the membrane potential gradient, we hypothesized that $\mathrm{JC} 15$ might be regulating the activity of channels, such as MPTP complex. MPTP complex is known to get activated under high ROS conditions and $\mathrm{Ca}^{2+}$ overload, ${ }^{13}$ leading to increase fluid uptake due to MPT; thus resulting in potential loss, organellar swelling and induction of cell death. ${ }^{13,17}$ As compared with control and JC15 $\mathrm{V}_{133 \mathrm{~W}}$ elevated levels of JC15 resulted in increased mitochondrial swelling upon exposure to $\mathrm{Ca}^{2+}$ due to activation of MPT (Figure 1e). Therefore, we hypothesized that matrix-oriented JC15 may be involved in the remodeling of MPTP activity through its matrix constituent CypD upon cisplatin treatment. CypD deletion or its inactivation by inhibitor cyclosporine $A$ (CsA) leads to development of cellular resistance to MPT. ${ }^{10,13,18}$ To test our hypothesis, we subjected JC15overexpressing cells to two alternative assays: first, pretreating the cells with CsA before cisplatin exposure; and second, depleting CypD followed by the drug treatment.

Upon pre-treatment with CsA, we observed $>50 \%$ suppression in drug sensitivity along with reversal of mitochondrial depolarization as indicated by the maintenance of potential (Figures $2 \mathrm{a}$ and $\mathrm{b}$ ), cytochrome $c$ release and caspase activity observed (Figure $2 \mathrm{c}$ and Supplementary Figure S6B) in JC15-overexpressing HEK293T cells and cancer cells (Supplementary Figures S3A-D, S5A and B). However, the significant release of cytochrome $c$ in cisplatin-treated cells even after CsA pre-treatment might probably be attributed to lower cellular retentive nature of CsA ( $<3 \mathrm{~h})$ and its non-specific binding to other cytosolic cyclophilins; ${ }^{20}$ hence, releasing CypD from its inhibited state and activating MPTP channel. To support our above observation, treatment of mitochondria with CsA attenuated the elevated swelling of the organelle under high JC15 levels, signifying restoration of membrane potential and integrity of the inner mitochondria membrane (Figure 1f).

We revalidated our above results using the second approach by depleting CypD under elevated JC15 levels in both non-cancerous and cancer-derived cells (Figure $3 a$ ). Downregulation of CypD exhibited rescue of cell viability in HEK293T (Figures 3b and c), OVCAR-3 (Supplementary Figures S4A and C) and MCF7 (Supplementary Figures S4B and D) cells expressing higher levels of $\mathrm{JC} 15$ upon cisplatin treatment. The cells also showed reduced levels of effecter caspase $3 / 7$ activity (Figure $3 d$ ) and a decline in the release of cytochrome $c$ (Figure $3 \mathrm{~g}$ ), indicating suppression of apoptosis. Concurrent to enhanced cell viability, JC15-overexpressing cells depleted for CypD showed mitochondrial repolarization by restoration of potential, demonstrated by inhibition of mitochondrial swelling (Figure 3f) and uptake of potential-sensitive MitoTracker dye (Figure 3e, Supplementary Figures S5A and B). The demonstration of a similar suppression of apoptosis phenotype by reduced expression of both JC15 and CypD suggested a possible functional connection in the common pathways.

Prevention of gain-of-function phenotype of CypD-overexpressing cells by JC15 knockdown. CypD overexpression is associated with consecutive MPTP opening and profound organellar swelling. Hence, to further substantiate the possible role of JC15 in activating MPTP through CypD, we contemplated whether lower levels of JC15 could rescue deleterious effects of elevated CypD. We overexpressed CypD alone or under reduced JC15 levels and analyzed for MPTP activation. Overexpression of CypD (Supplementary Figure S2B) resulted in gain-of-function effects on MPTP by promoting constitutive $\mathrm{MPT}^{18}$ even under untreated
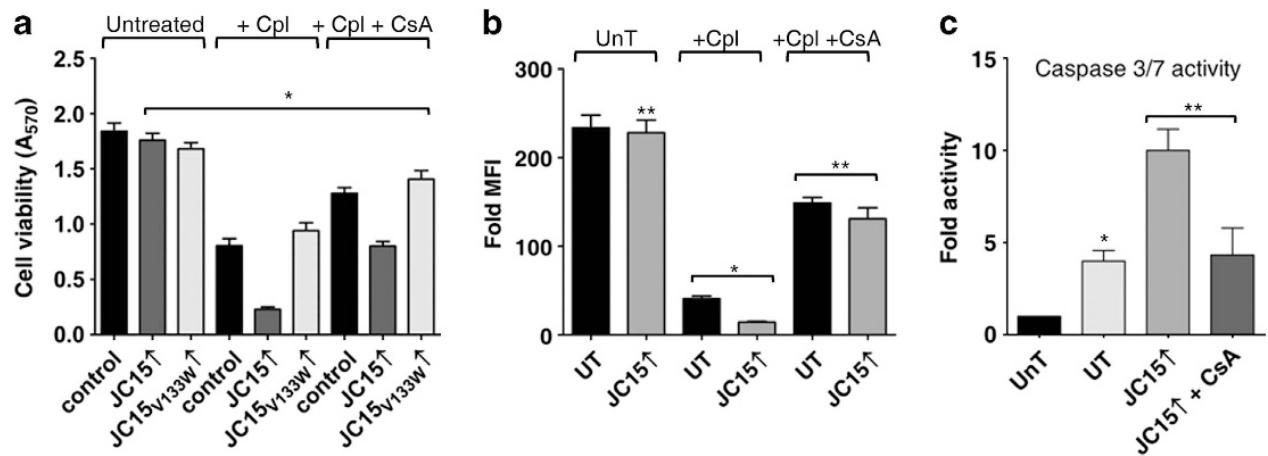

Figure 2 CsA inhibits chemosensitive phenotype of JC15-overexressing cells. (a) Cells were left untreated, treated with cisplatin ( + Cpl) alone or supplemented with $1 \mathrm{nM}$ $\mathrm{CsA}(+\mathrm{Cpl}+\mathrm{CsA})$ and subjected to MTT assay. Data shown as mean \pm S.E.M. $n=8$, ${ }^{*} P$ (two tailed) $<0.0001$. (b) Cells expressing wild-type JC15 were left untreated $($ UnT), pre-treated with CsA followed by cisplatin exposure $(+\mathrm{Cpl}+\mathrm{CsA})$ or treated with cisplatin alone $(+\mathrm{Cpl})$. The opening of mitochondrial transition pore was adjudged by staining with potential-sensitive MitoTracker Red CMXRos dye. UT, untransfected cells; UnT, untreated cells. Data are represented as mean \pm S.E.M. $n=3$, ${ }^{*} P$ (two tailed) $<0.0001,{ }^{* *} P$ (two tailed) $<0.001$. (c) Relative caspase activity of cisplatin-treated JC15-expressing cells pre-exposed to CsA represented as mean \pm S.E.M. $n=3$, ${ }^{\star} P$ (two tailed) $<0.0001,{ }^{* \star} P$ (two tailed) $<0.001$ 

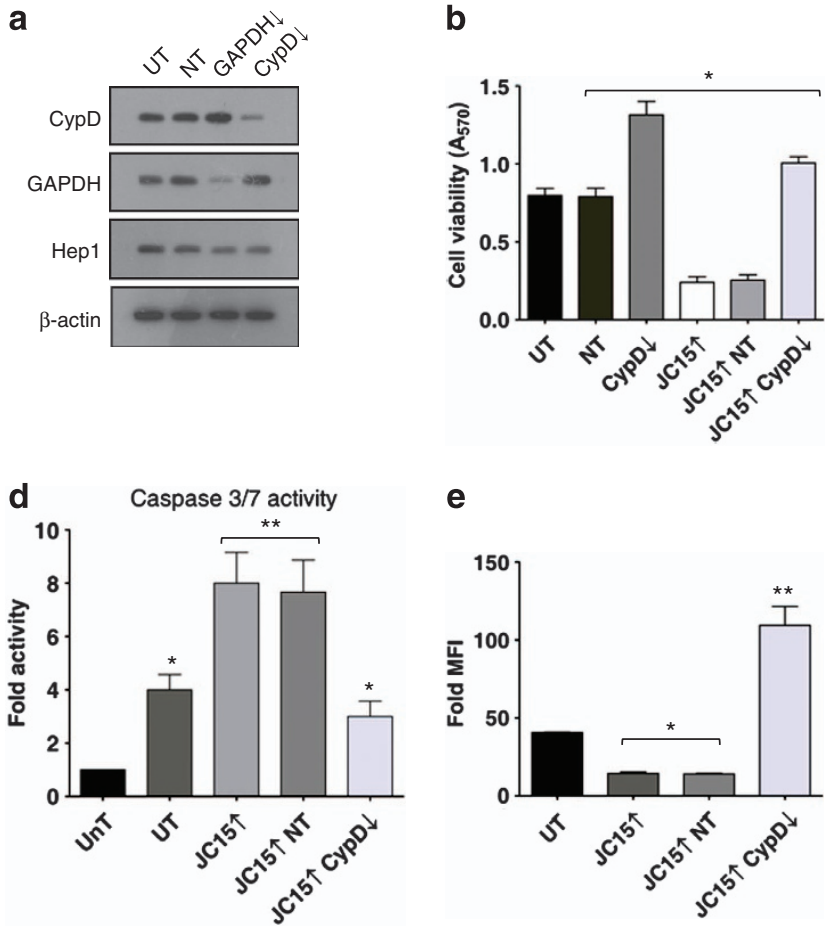

e

C
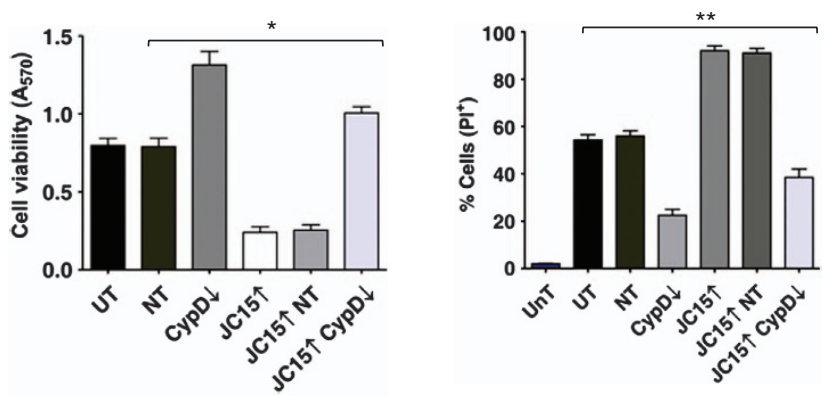

f

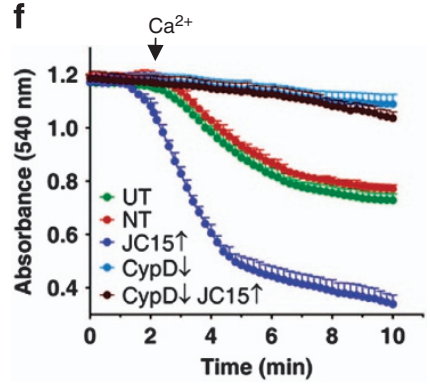

g

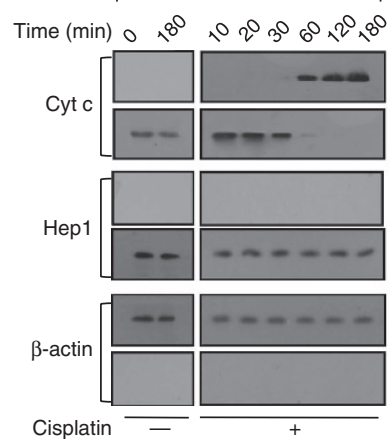

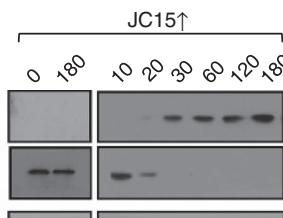
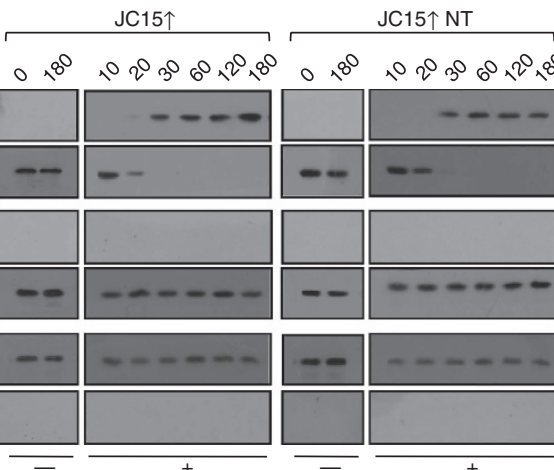
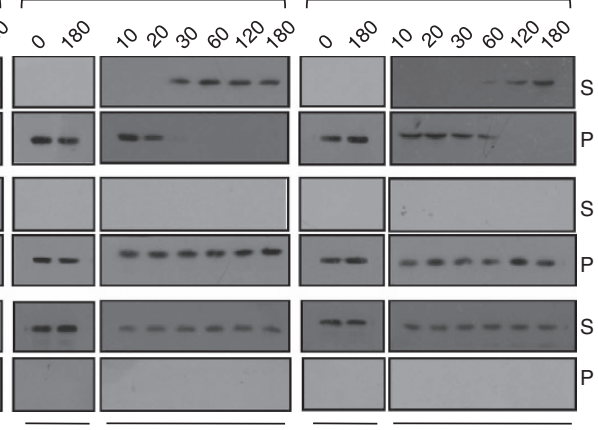

Figure 3 Modulation of JC15-mediated chemosensitivity by CypD. (a) Expression blot of HEK293T cells transfected with siRNA pool against CypD. (b) Mortality of cisplatin-treated cells with variable levels of CypD, and JC15 was determined using MTT assay. Bars represent mean \pm S.E.M. $n=8$, ${ }^{*} P$ (two tailed) $<0.0001$. (c) Cell mortality of cisplatin-treated cells as mentioned in panel (b), was determined by propidium iodide (PI) uptake. Data shown as mean \pm S.E.M. $n=3,{ }^{* \star} P$ (two tailed) $<0.001$. (d) Relative caspase activity of cisplatin-treated JC15-expressing cells depleted for CypD, represented as mean \pm S.E.M. $n=3,{ }^{*} P$ (two tailed) $<0.0001,{ }^{* *} P$ (two tailed) $<0.001$. (e) The opening of mitochondrial transition pore was determined by staining with potential-sensitive dye MitoTracker Red. Acquired data are represented as fold mean florescence intensity (MFI) over unstained cells and shown as mean \pm S.E.M. $n=3,{ }^{\star} P$ (two tailed) $<0.0001$, ${ }^{\star \star} P$ (two tailed) $<0.001$. (f) Mitochondria isolated from CypD-depleted cells under normal or JC15-overexpressed conditions was subjected to swelling. The absorbance is shown as mean \pm S.E.M. $n=3, P<0.0001$ with reference to UT. (g) HEK293T cells differentially expressing JC15 and CypD were exposed to cisplatin treatments for the indicated time intervals. Post treatment, the isolated mitochondria $(P)$ along with the cytosolic supernatant $(S)$ were subjected to immunoblot analysis using an anti-cytochrome $c$ (Cytc) antibodies. For all the above experiments, untreated (UnT), untransfected cells (UT) and non-targeting siRNA-treated cells (NT) were used as internal negative control. ( $\downarrow$-denotes mRNA downregulated by dsiRNA, $\uparrow$-denotes overexpression)

conditions. Exposure of cells with increased CypD levels to cisplatin resulted in reduced cell viability (Figures $4 a$ and b; Supplementary Figures S4E-H), cytochrome $c$ release (Figure 4e) and an increase in caspase activity (Figure 4f). The cells also showed a significant degree of mitochondrial potential loss (Figure 4c) and pronounced intrinsic baseline swelling of mitochondria even in the absence of $\mathrm{Ca}^{2+}$ (Figure 4d) in both 293T and cancer cells (Supplementary Figures S5C and D). The pro-apoptotic phenotype of elevated CypD was significantly nullified by downregulating JC15.
The cells showed better survivability (Figures $4 a$ and b), reduced caspase activity (Figure $4 \mathrm{f}$ ) and contained significant amounts of repolarized mitochondria (Figure 4c), together with marked reduction in mitochondrial swelling (Figure 4d) and inhibition of cytochrome $c$ release (Figure $4 \mathrm{e}$ ) in HEK293T and cancer cells (Supplementary Figures S4E-H, S5C and D). This demonstrates the existence of a functional interaction between CypD and JC15. CypD overexpression leads to its disregulated recruitment to the MPTP channel causing constitutive pore opening and dynamic swelling of 
a

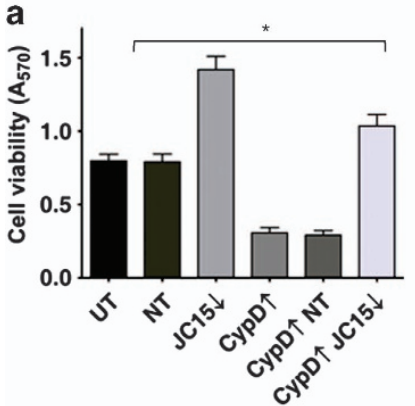

e

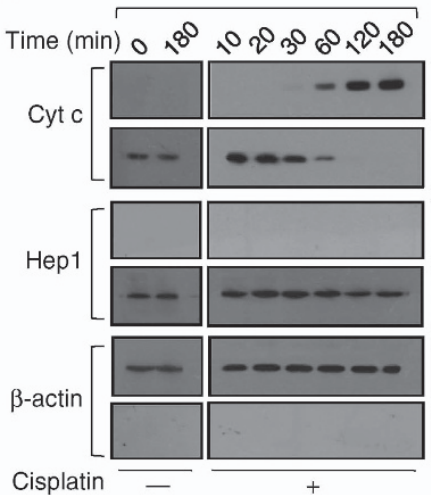

f

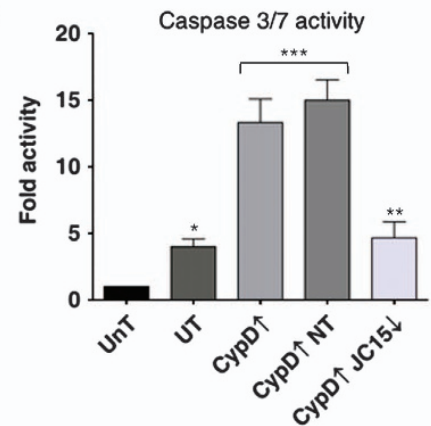

b

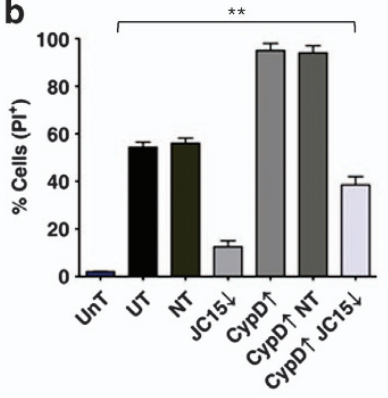

C

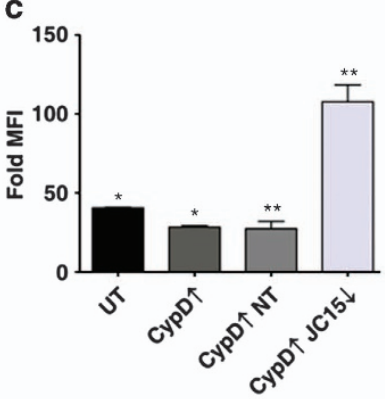

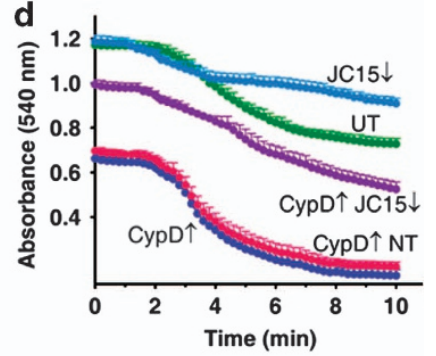
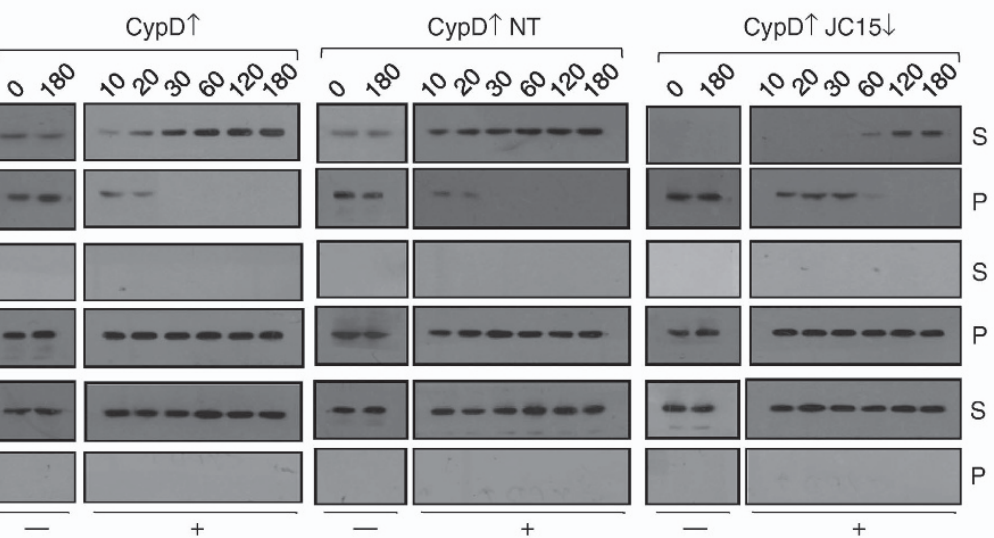

g
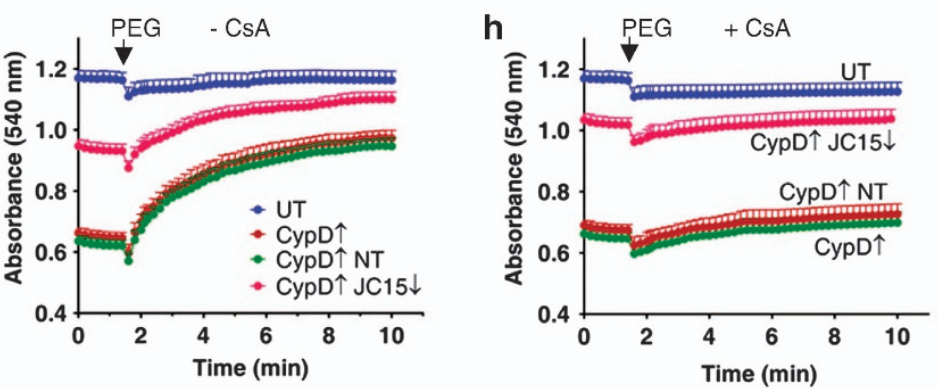

Figure 4 Suppression of elevated CypD phenotypes by JC15 depletion. (a) Relative cell viability measurement of cisplatin-treated cells under differential levels of CypD and JC15 by MTT assay. Data are represented as mean \pm S.E.M. $n=8,{ }^{*} P$ (two tailed) $<0.0001$. (b) Relative cell death of cisplatin-exposed cells differentially expressing CypD and JC15 was assayed through PI staining. Bars represent mean \pm S.E.M. $n=3,{ }^{* *} P$ (two tailed) $<0.001$. (c) Cisplatin-treated cells overexpressing CypD under normal or reduced JC15 protein levels were stained with MitoTracker and represented as fold mean florescence intensity (MFI) over unstained cells. Data shown as mean \pm S.E.M. $n=3,{ }^{*} P$ (two tailed) $<0.0001,{ }^{*} P$ (two tailed) $<0.001$. (d) Mitochondria expressing altered levels of indicated proteins was subjected to swelling, and the decrease in absorbance was represented as mean \pm S.E.M. $n=3, P<0.0001$. (e) Untransfected (UT) or transfected cells with altered amounts of CypD or JC15 were treated with cisplatin for the indicated time periods and assayed for Cytc release as described in Figure $2 \mathrm{~g}$. (f) Relative caspase activity of cisplatin-treated cells containing altered levels of CypD and JC15 represented as mean \pm S.E.M. $n=3,{ }^{*} P$ (two tailed) $<0.0001,{ }^{* *} P$ (two tailed) $<0.001,{ }^{* * *} P<0.01$. UnT, untreated cells; UT, untransfected treated cells. ( $g$ and $\mathbf{h}$ ) Mitochondria overexpressing CypD alone or with reduced amounts of JC15 were induced to shrink with $5 \%$ PEG-3340 after pre-treating with $1 \mathrm{nM}$ cyclosporin $\mathrm{A}$ (h) or were left untreated (g). Data are shown as mean \pm S.E.M. $n=3, P<0.0001$

the mitochondria, which can be further assessed by PEGdependent mitochondrial shrinkage. PEG treatment alleviated the pronounced baseline swelling of unsilenced mitochondria and partially swelled condition of mitochondria with reduced levels of JC15 (Figure $4 \mathrm{~g}$ ), which was, however, inhibited by pre-treatment with CsA (Figure 4h). This indicates that swelling was specifically induced due to recruitment of CypD to MPTP, which is largely governed by the expression levels of JC15. In summary, the above results indicate that JC15 might be involved in maturation of MPTP complex through association of CypD in both normal and cancer cells.
Interdependence of JC15 and CypD in MPTP activation and its consequent effects in live cells. To demonstrate a similar CypD-dependent modulation of MPT by JC15 in live cells, we subjected MCF7 cells, differentially expressing $\mathrm{JC} 15$ and $\mathrm{CypD}$, to $\mathrm{CoCl}_{2}$-calcein fluorescence quenching assay. ${ }^{21-23}$ Upon cisplatin treatment, the calcein fluorescence in JC15-overexpressing cells was found to be significantly lower than untransfected controls, suggesting an increase in MPTP opening (Figure 5b). In contrast, both JC15-depleted and $\mathrm{JC} 15_{\mathrm{V} 133 \mathrm{~W}}$-overexpressing cells had higher calcein fluorescence than control cells (Figures $5 \mathrm{a}$ and b). Depletion of CypD or its inhibition by CsA in JC15-overexpressing 

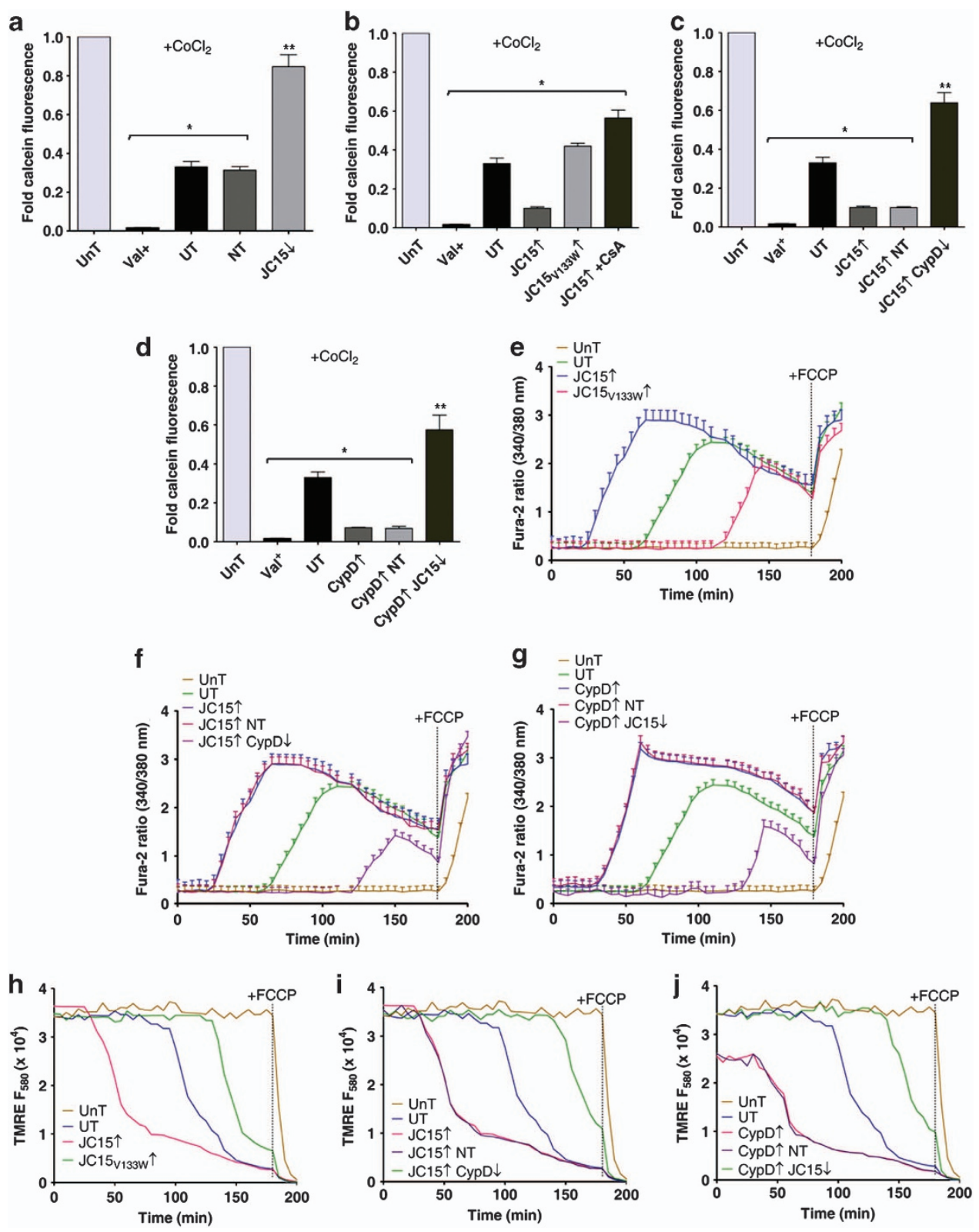

Figure $5 \mathrm{JC} 15$ induces permeability transition through CypD in live cells. (a) MCF7 cells depleted for $\mathrm{JC}_{15}$ were exposed to cisplatin treatment and subjected to $\mathrm{CoCl}_{2}$ calcein quenching assay. The amount of calcein fluorescence was quantified by flow cytometry and is represented in the form of bar chart as mean \pm S.E.M. $n=3$, ${ }^{\star} P<0.0001$, ${ }^{*} P<0.001$. (b and $\left.\mathbf{c}\right) \mathrm{JC} 15$ overexpressing cells either pre-treated with CsA (B) or depleted for CypD (C) were loaded with calcein dye before quenching by $\mathrm{CoCl}_{2}$. The relative calcein emission was quantified by flow cytometry and represented as fold fluorescence with respect to cisplatin-untreated cells. Data denote mean \pm S.E.M. $n=3,{ }^{*} P<0.0001,{ }^{* *} P<0.001$. (d) The opening of MPTP under elevated CypD in the presence of normal or downregulated levels of JC15 was quantified by relative quenching of calcein dye and plotted as mean \pm S.E.M. $n=3,{ }^{*} P<0.0001,{ }^{*} P<0.001$. (e-g) Time-dependent increase in cytosolic calcium levels due to MPTP opening, following cisplatin treatment of MCF7 cells with differential protein expressions, was measured by spectrofluorometric analysis of Fura-2 excitation. Data denote mean \pm S.E.M. $n=3, P<0.01$ with reference to UnT cells. (h-j) Time course analysis of the relative propensity of membrane potential loss due to MPT in cells with variable levels of JC15 and CypD was performed using TMRE uptake. The line graphs represented mean values from three independent experiments. (UnT, untransfected untreated cells; UT, untransfected treated cells; NT, cells transfected with non-targeting siRNA; $\downarrow$-denotes downregulation by siRNA; $\uparrow$ — denotes overexpression; Valinomycin (Val) and FCCP were used as validation controls for dissipation of membrane potential and intracellular calcium increase)

background restored the calcein signal, indicative of the fact that $\mathrm{JC} 15$ might be mediating MPTP opening through CypD (Figures $5 \mathrm{~b}$ and $\mathrm{c}$ ). Finally, we quantified the calcein fluorescence in CypD-overexpressing cells, which was considerably lower than the controls. This decrease in fluorescence was, however, reversed upon depletion of JC15 (Figure 5d). The calcein fluorescence, in the absence of $\mathrm{Co}^{2+}$, was similar in both control and depleted cells, indicating equivalent calcein loading (Supplementary Figure 7). This shows that JC15 mediates MPTP activation through CypD and thus promotes increased sensitivity of cells to chemotherapeutic drugs.

Dynamic changes in the intracellular calcium levels in response to various stimuli are known to regulate different 
cellular processes, including apoptosis. ${ }^{24}$ Elevated mitochondrial calcium levels are known to induce opening of the MPTP, leading to release of pro-apoptotic factors. ${ }^{25,26}$ Therefore, we performed real-time measurements of mitochondrial calcium levels under depleted conditions when the cells were exposed to cisplatin. Opening of MPTP will allow the cations to freely redistribute across the membranes according to their concentration gradients, which can be monitored through Fura-2 stain. Cells overexpressing JC15 showed higher enhancement in Fura-2 signal comparatively earlier than untransfected controls. On the other hand, cells expressing JC15 mutant showed resistance to mitochondrial calcium release (Figure 5e). The increment in a Fura-2 ratio observed in case of JC15 upregulation was substantially reduced upon depletion of CypD, highlighting an association of JC15 with MPT upon cisplatin treatment (Figure 5f). Overexpression of CypD made the cells susceptible to MPTP activation, resulting in a sharp increase in cytosolic calcium levels, which was alleviated upon depletion of JC15 (Figure $5 \mathrm{~g}$ ), underlying the dependence of CypD on JC15 for opening of MPTP channel. As a control, the specificity of calcium discharge due to pore formation was determined by treating the cell with protonophore FCCP, which is known to cause enhancement in intracellular calcium release.

To further validate that the observed calcium flux is due to MPTP channel opening, we monitored the alterations in mitochondrial potential real-time by utilizing tetramethylrhodamine ethyl ester (TMRE) fluorophore, which reversibly diffuses into mitochondria in a potential-dependent manner. Conceivably, upregulation of both $\mathrm{JC} 15$ and CypD resulted in a sharp decrease in membrane potential, which overlapped with the mitochondrial calcium release (Figures $5 \mathrm{~h}-\mathrm{j}$ ). The decline in membrane potential was, however, found to be protected under CypD or JC15 depletion, respectively (Figures 5i and j). Similarly, cells expressing JC15 mutant showed higher membrane potential than untransfected cells (Figure 5h). FCCP as a positive control completely dissipated the membrane potential, thus adding specificity to the reaction. In summary, our results emphasize the interplay between JC15 and CypD in regulation of MPT, thereby modulating cellular resistance to cisplatin treatment.

Loss-of-membrane selectivity across the mitochondrial inner membrane for solutes leads to ionic imbalance and dissipation of the potential gradient. This results in uncoupling of electrons from electron transport chain complexes, which in turn react with molecular oxygen to give rise to free radicals. As both JC15 and CypD overexpression cause activation of MPTP channel, we assessed whether they are associated with concomitant increase in ROS levels. The overall ROS was quantified in the cells by DCFDA-H2 staining and mitochondrial superoxide levels by labeling the cells with MitoSOX. Cisplatin treatment of cells containing elevated levels of JC15 or CypD showed intense staining of both DCFDA and MitoSOX (Figures $6 a-d)$. The relative fluorescence of the dyes was, however, reduced upon downregulating JC15 under CypD-overexpressed conditions (Figures $6 c$ and $d$ ). Similar observation was recorded for JC15-overexpressing cells that were silenced for CypD, and the cells depicted lesser oxidation of both DCFDA and MitoSOX dyes (Figures $6 a$ and $b$ ). These observations further validate coupling of $\mathrm{JC} 15$ and $\mathrm{CypD}$ functions in the activation of MPTP complex.

Recruitment of CypD to the MPTP channel is mediated by JC15. The inhibition of gain-of-function effects of elevated CypD leading to constitutive MPTP activation by JC15 depletion suggested a role of JC15 in recruitment of CypD to the MPTP channel. To prove direct recruitment of CypD into translocase in JC15-overexpressed condition, we performed CoIP analysis utilizing HEK293T mitochondria under cisplatin-treated and -untreated condition. Upon cisplatin treatment, we observed increased recruitment of CypD along with its matrix interacting Hsp90 class protein, TRAP $1^{27,28}$ as compared with untransfected controls (Figure 6f; panel 1) when ColP was performed using antiJC15 antibodies. Similarly, ColP using anti-CypD antibodies under high JC15 levels resulted in increased immunoprecipitation of JC15 and mitochondrial heat-shock protein 70 (mtHsp70) but reduced levels TRAP1 in immunoprecipitated complexes as compared with untransfected cells (Figure 6f; panel 2). However, no pull-down of either CypD/TRAP1 or mtHsp70/JC15 was observed in case of untreated cells (Figure 6f) and JC15 or CypD downregulated cells for respective ColP experiments (Figure $6 f$ ). To further validate our point, we subjected $\mathrm{JC} 15_{\mathrm{V} 133 \mathrm{w}}$-overexpressing mitochondria to ColP analysis under cisplatin-treated and untreated conditions. Absence of the co-immunoprecipitating band of CypD and TRAP1 in mutant cells is indicative of the specific role played by $\mathrm{JC} 15$ in recruiting CypD to MPTP complex and critical role of a functional J-domain in mediating such interaction (Figure 6e). The recruitment of CypD to the MPTP channel through its interaction with JC15 was further verified in cancer-derived MCF7 cells. Consistent with above results, overexpressed JC15 was associated with increased amounts of CypD (Figure $6 \mathrm{~g}$ ), and the interaction was mediated by its J-domain (Figure 6h). In summary, our results highlight the importance of $\mathrm{JC} 15$ in recruitment of CypD to the MPTP complex and thereby inducing apoptosis through permeability transition.

\section{Discussion}

Mitochondrial J-proteins have been primarily known to have a critical role in protein translocation and folding reaction in the organelle. JC15, belonging to type III class of J-protein, is an ortholog of yeast Pam18 and have an important role in transport of precursor proteins in the mitochondria. At the translocation channel, JC15 is involved in stimulation of mtHsp70 ATPase activity for efficient import of precursor proteins. However, the lower expression of JC15 associated with chemorefractory cancer cells is indicative of its potential role in regulation of apoptosis. The percentage of the $\mathrm{CpG}$ island methylation induced by chemotherapeutic drugs not limited to cisplatin, taxols and doxorubicin, directly co-related with the relative $\mathrm{JC} 15$ gene expression and cellular drug response in tumor sub-population. ${ }^{29}$ These results suggest JC15 expression profile to be a key determinant of cell death mechanism. However, further work is needed to elucidate the molecular mechanism of drug-induced methylation of JC15 promoter in tumor samples. In the present study, we propose 

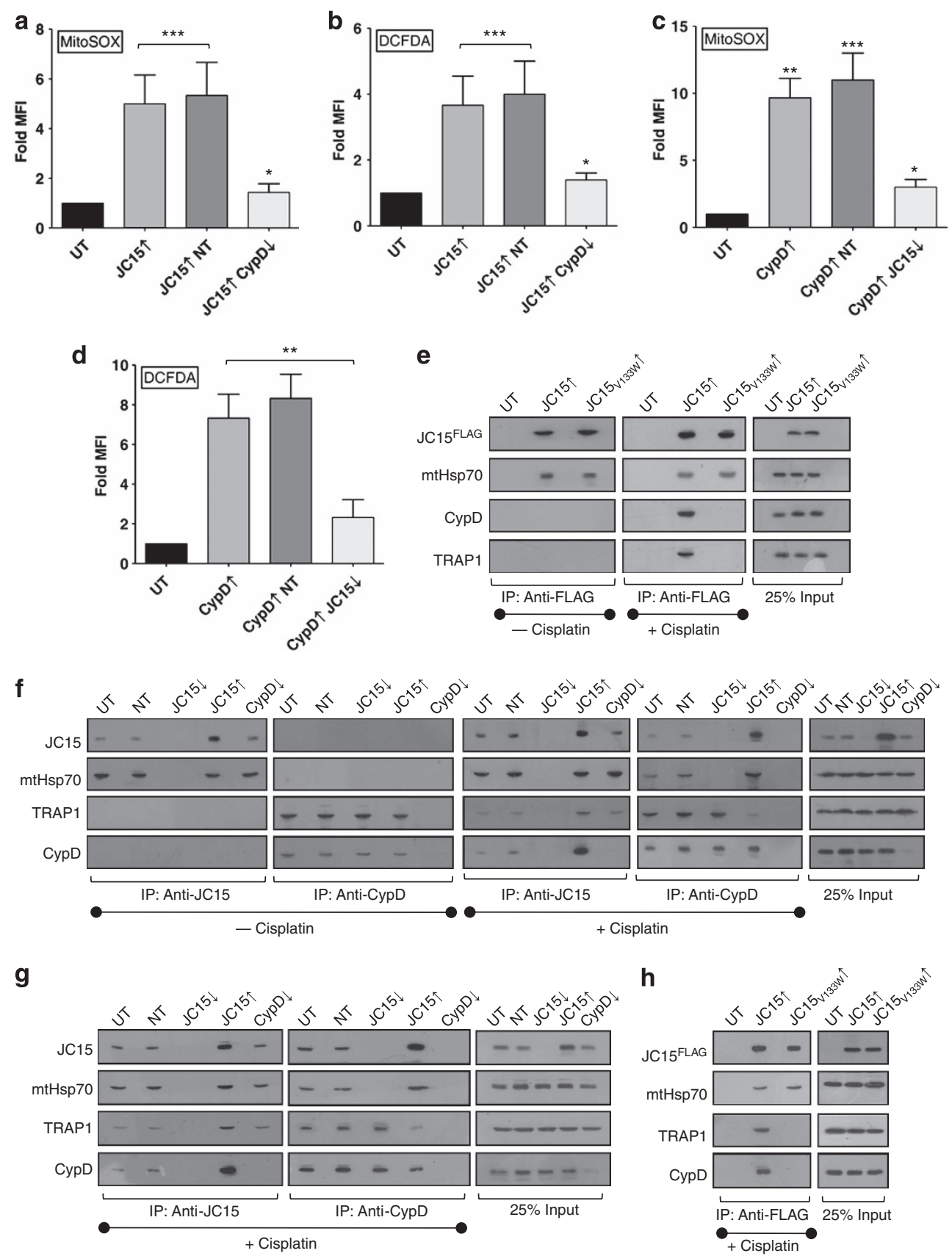

Figure 6 Coupling of CypD activation with permeability transition by JC15. (a-d) The relative alterations in mitochondrial superoxide and cellular ROS upon cisplatin treatment, in cells with variable JC15 and CypD expression were quantified by MitoSOX and DCFDA staining as indicated. Bars represent mean \pm S.E.M. $n=3$, ${ }^{*} P<0.0001$, ${ }^{* *} P<0.001,{ }^{* * *} P<0.01$. (e and f) Mitochondria isolated from cisplatin-treated or -untreated HEK293T cells expressing altered levels of wild-type JC15, JC15 ${ }_{\mathrm{V} 133 \mathrm{~W}}$ or CypD were lysed in $0.5 \%$ digitonin buffer in the presence of $5 \mathrm{mM}$ EDTA, followed by ColP and immunodecoration with specific antibodies. ( $g$ and $\mathbf{h}$ ) The above ColP experiment was repeated in MCF7 cells containing variable levels of wild-type $\mathrm{JC} 15, \mathrm{JC} 15_{\mathrm{V} 133 \mathrm{~W}}$ or CypD. (UnT, untreated; UT, untransfected; NT, non-targeting siRNA; $\downarrow-$ denotes mRNA downregulated by dsiRNA, $\uparrow$-denotes overexpression)

an additional function of $\mathrm{JC} 15$ in regulating cell death effectors, thereby modulating the sensitivity of cells to chemotherapeutic drugs.

Although many of the components of human presequence translocase such as Tim17a, Magmas and JC15 are variably expressed in the subset of cancer cells, ${ }^{7,8,29-31}$ obtaining a direct link between dynamic restructuring of translocation machinery leading to stimulation of cellular death machinery was obscure. Here, for the first time, we provide evidence in favor of specific activation of translocase upon xenobiotic treatment. JC15 as a part of translocase alters the apoptogenic stimuli by modulating the functionality of MPTP complex through CypD. CypD belongs to cyclophilin class of immunophilins and localizes in the mitochondrial matrix where it has an essential role in activating MPTP channel to cause MPT. ${ }^{10,18}$ Short-circuiting of ionic balance due to MPT results 
in increased organellar swelling leading to outer membrane disruption and release of pro-apoptotic factors. ${ }^{10,13}$ However, the exact mechanistic details of the overall process are still unknown. Our data suggest that elevated JC15 cause MPTP activation, thereby resulting in enhanced calcium levels, mitochondrial potential loss, organellar swelling and release of death effectors. On the other hand, reduced amounts of JC15 protects against MPT and mitochondrial depolarization even under high levels of CypD, which is known to cause constitutive opening of MPTP. This indicates toward the definitive role of $\mathrm{JC} 15$ in coupling of CypD to the MPTP channel. Besides, our results also demonstrate the critical role of JC15's J-domain in its association with CypD and induction of MPT. It is as well possible that, apart from interacting with CypD, a functional J-domain carrying out proper import activity is essential for mounting a chemosensitive response, as previous studies have hypothesized loss of import motor activity in the development of oncocytic tumors. ${ }^{32}$

Based on our results, we provide a model illustrating the specific role played by $\mathrm{JC} 15$ in conjunction with $\mathrm{mtHsp70}$ to promote membrane pore transition by activating MPTP complex through CypD (Figure 7). In cancer cells, CypD remains in the inactive state by directly interacting with chaperones, such as TRAP1 (mitochondria localized Hsp90 class of protein). ${ }^{27}$ Overexpression of TRAP1 in tumor cells and its increased mitochondrial localization lead to CypD inactivation, thus causing the cells to propagate with a suppressed MPTP function. ${ }^{27,33}$ In our model, we demonstrate stress-responsive recruitment of $\mathrm{CypD}$ and its concomitant release from TRAP1 by JC15-mtHsp70 complex. Release of CypD from TRAP1 results in its activation and is, in turn, coupled with the MPTP complex by JC15-mtHsp70 machinery (Figure 7). This results in pore opening and induction of cell death; partly explaining the chemoresistant phenotype observed in JC15-depleted cancer cells. ${ }^{7,8,19,29}$

In general, mitochondria have a central role in apoptosis and cancer and have been one of the prime therapeutic targets to control cancer progression. Mitochondrial multichaperone interactor network has evolved as nodal points in key regulatory circuits, generating an interesting grid for neoplastic studies. Identification of protein translocase component JC15's association with oncocytic development is one of the cornerstones of the mitochondrial cell death pathways. This will provide a directed approach in understanding the dynamic scheme of interaction in carcinogenesis and the role of mitochondrial chaperones in the process. The current work presents a unique connection between human presequence translocase and apoptosis regulator MPTP complex. Our study provides the first evidence for the involvement of translocation machinery in regulation of apoptotic pathways in cells subjected to chemotherapeutic treatment.

\section{Materials and Methods}

Cells and cell culture. HEK293T, OVCAR-3 and MCF7 cells were cultured in Dulbecco's modified Eagle's medium containing $10 \%$ fetal bovine serum and $1 \%$ penicillin-streptomycin. Transfection of the cells with the expression constructs was carried out using Lipofectamine 2000 (Invitrogen, Carlsbad, CA, USA) as per the manufacturer's instructions and allowed to express for $48 \mathrm{~h}$. All the cell lines were obtained from National Center of Cell Science, Pune, India repository. The cell lines were authenticated through standard STR-based profiling before the experiments.

Plasmids. The expression of DNAJC15 or CYCLOPHILIN D in human cell lines was carried out by amplification of the C-terminal FLAG-tagged ORF of DnaJC15 or CypD and cloning it in $\mathrm{pCl}-\mathrm{Neo}$ vector. The loss-of-function J-domain mutant was subsequently generated through QuikChange site-directed mutagenesis protocol (Stratagene, La Jolla, CA, USA).

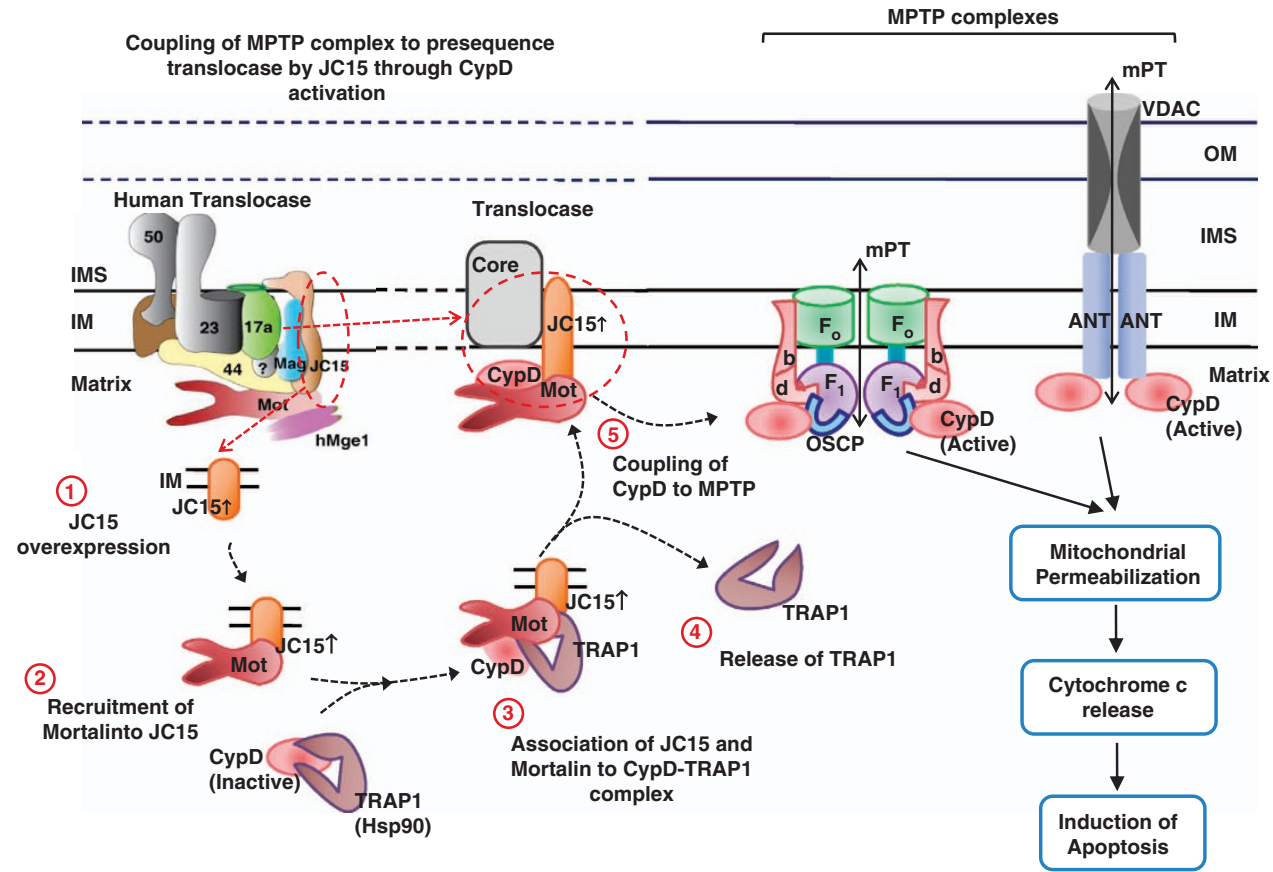

Figure 7 Model illustrating the mechanism of mitochondrial transition pore regulation by JC15. JC15 mediates MPT through CypD activation and its coupling to the MPTP complex under chemotherapeutic treatment. The model represents both forms of MPTP complex: the traditional model composed of VDAC, ANT and CypD; and the recently proposed model comprising of $\mathrm{F}_{0}-\mathrm{F}_{1}$ dimers, where CypD associates with the OSCP subunit 
Antibodies and immunoblotting. For expression analysis of DnaJC15 in yeast, equivalent amounts of cells were lysed and analyzed by western blotting using anti-DnaJC15 antibodies. The expression analysis of proteins in human mitochondria was performed by analyzing $50 \mu \mathrm{g}$ of mitochondrial protein by immunodecorating with specific antibodies. The following antibodies were used for primary detection: anti-Tim23 $(1: 5000)$, anti-CypD $(1: 1000)$ (Pierce Thermo Scientific, Rockford, IL, USA), anti-cytochrome c (1:1000) (Cell Signaling Technology, Danvers, MA, USA); anti-DnaJC15 antibodies generated by injecting their corresponding J-domain fragments; anti-mortalin (mtHsp70) antibodies were developed against the C-terminal region; and anti-Hep1 was raised against the full-length protein at Imgenex Biotech (Bhubaneshwar, India). Anti-TRAP1 antibodies were a kind gift from Professor Didier Picard, Department of Cell Biology, University of Geneva, Geneva, Switzerland. For co-immunoprecipitation analysis, antisera were affinity purified and cross-linked to either Protein $A$ or Protein $G$ beads (GE Healthcare, Amersham, UK). Secondary immunodecoration was performed using HRP-conjugated anti-rabbit or anti-mouse IgG (GE Healthcare).

RNAi knockdown experiments. HEK293T, OVCAR-3 or MCF7 cells were seeded in Opti-MEM media (Invitrogen) and transfected with $5 \mu \mathrm{M}$ of DsiRNA duplexes pools; ON-TARGETplus SMART pool (Dharmacon, Lafayette, CO, USA) for DNAJC15; HSC.RNAI.N005038.12.1 and HSC.RNAI.N005038.12.2 (IDT) for CYCLOPHILIN D. Transfection was performed using Lipofectamine 2000 (Invitrogen) using the manufacturer's instructions.

Analysis of cellular sensitivity in human cell lines. Untransfected and transfected HEK293T cells were exposed to $10 \mu \mathrm{M}$ cisplatin for $3 \mathrm{~h}$ unless otherwise mentioned. Relative cell death coupled with mitochondrial dysfunction was determined by AnnexinV--AlexaFlour 488/propidium iodide and AnnexinVAlexaFlour 488/MitoTracker Red CMXRos (Invitrogen) staining. The loss of membrane potential alone was determined by loading the cells with MitoTracker Red CMXRos at $37^{\circ} \mathrm{C}$. The relative fluorescence was analyzed by BD FACS Canto II flow cytometer (San Jose, CA, USA) using the BD FACS Diva software. The time lapse analysis of membrane potential loss was performed by staining the cells by TMRE (Molecular Probes; Invitrogen, Eugene, OR, USA) and measuring the relative variation in fluorescence at $580 \mathrm{~nm}$ using Tecan Infinite M1000 PRO spectofluorometer (Männedorf, Switzerland).

Cell viability and cell proliferation. The percentage of viable cells was determined by 3-(4,5-Dimethylthiazol-2-yl)-2,5-diphenyltetrazolium bromide assay (MTT assay) (Invitrogen) as instructed in the manufacturer's manual. The MTT assay data were further verified by flow cytometric analysis of relative propidium iodide uptake by the cells. The cells with positive propidium iodide staining signified the dead cells. The induction of apoptosis was determined by measuring the relative effecter caspase activity using the Caspase-Glo 3/7 Assay kit (Promega, Madison, WI, USA) following the manufacturer's instructions.

Mitochondrial swelling and cytochrome $c$ release assay. The opening of mitochondrial transition pore complex was determined by mitochondrial swelling and shrinking assay. ${ }^{18}$ In all, $130 \mu \mathrm{g}$ mitochondria was resuspended in $500 \mu \mathrm{l}$ of assay buffer and subjected to swelling induced by $250 \mu \mathrm{M} \mathrm{CaCl}$. Mitochondrial shrinkage was promoted by incubating the mitochondria in buffer containing $5 \%(\mathrm{w} / \mathrm{v})$ PEG-3340. Temporal variation in light scattering was measured at $540 \mathrm{~nm}$. For cytochrome $c$ release assay, cells were exposed to cisplatin for different time intervals, and the corresponding mitochondrial and cytosolic fractions were assayed for the presence of cytochrome $c$ through immunoelectrophoresis. In all, $120 \mathrm{mM} \mathrm{KCl}, 10 \mathrm{mM}$ Tris pH 7.6 and $5 \mathrm{mM} \mathrm{KH}_{2} \mathrm{PO}_{4}$ was used as the assay buffer for both swelling and cytochrome $c$ release experiments.

Assaying MPTP opening in live cells. The determination of MPT in live cells was carried out using $\mathrm{CoCl}_{2}$-calcien quenching assay. Calcein-AM is a membrane permeable fluorophore that diffuses to all subcellular compartments, including mitochondria. The cells are then loaded with divalent cobalt cation $\left(\mathrm{Co}^{2+}\right)$, which can quench calcein fluorescence from all subcellular compartments except mitochondrial matrix owing to the impermeability of inner mitochondrial membrane to $\mathrm{Co}^{2+}$ cations. However, opening of MPTP causes $\mathrm{Co}^{2+}$ to diffuse inside mitochondria and quench the mitochondrial calcein fluorescence. Cisplatintreated or -untreated MCF7 cells were loaded with $1 \mu \mathrm{M}$ Calcein-AM dye and
$100 \mathrm{nM}$ MitoTracker Deep Red for $30 \mathrm{~min}$ at $37^{\circ} \mathrm{C}$. The calcein fluorescence was quenched by adding $1 \mathrm{mM} \mathrm{CoCl} 2$ for $10 \mathrm{~min}$ and subjected to flow cytometric analysis using BD FACS Canto II. Data were analyzed using the BD FACS Diva software. Valinomycin treatment was used as a positive control.

Analysis of mitochondrial calcium release. The analysis of mitochondrial calcium release was performed by adapting the previously described method. ${ }^{34}$ Briefly, MCF7 cells were stained with Fura-2 AM (Molecular Probes; Invitrogen) for $45 \mathrm{~min}$ at $37^{\circ} \mathrm{C}$ before cisplatin treatment. The temporal variation in Fura-2 fluorescence at $340 / 380 \mathrm{~nm}$ excitation upon mitochondrial calcium release was quantified using Tecan Infinite M1000 PRO spectofluorometer. Trifluorocarbonylcyanide phenylhydrazone (FCCP) was used as a positive control.

Measurement of cellular ROS levels. The elevation of cellular ROS levels, owing to MPT, was quantified by staining the cells with MitoSOX or DCFDA (Molecular Probes; Invitrogen) after $3 \mathrm{~h}$ cisplatin treatment. MitoSOX provided a measure of mitochondrial superoxide levels, and DCFDA quantified the overall cellular ROS levels. The relative fluorescence of the dyes was quantified by flow cytometry.

Co-immunoprecipitation analysis. The interaction between $\mathrm{JC} 15$ and CypD was determined by co-immunoprecipitation analysis using isolated mitochondria. Mitochondria from HEK293T cells were isolated using Cell Mitochondria Isolation Kit (Sigma, St. Louis, MO, USA). Mitochondria were lysed using $0.5 \%$ digitonin in a lysis buffer $(20 \mathrm{mM}$ MOPS-KOH pH 7.4, $250 \mathrm{mM}$ sucrose, $80 \mathrm{mM} \mathrm{KCl}$ and $1 \mathrm{mM}$ PMSF) containing $5 \mathrm{mM}$ EDTA. Lysates were subjected to immunoprecipitation analysis using antibodies specific to JC15, CypD or FLAG-tag as per requirement.

\section{Conflict of Interest}

The authors declare no conflict of interest.

Acknowledgements. We thank the Flow Cytometry facility of the Indian Institute of Science, Bangalore for flow cytometry experiments. We are obliged to Professor Didier Picard, Department of Cell Biology, University of Geneva for antiTRAP antibodies. We are also thankful to Miss Shubhi Srivastava and Mrs. Lekshmi Krishna for their help in performing experiments. This work was supported by funding from the DST-Swarnajayanthi fellowship scheme (to PDS) and Council of Scientific and Industrial Research Fellowship (to DS).

1. Kampinga HH, Craig EA. The HSP70 chaperone machinery: J proteins as drivers of functional specificity. Nat Rev Mol Cell Biol 2010; 11: 579-592.

2. Kozany C, Mokranjac D, Sichting M, Neupert W, Hell K. The J domain-related cochaperone Tim16 is a constituent of the mitochondrial TIM23 preprotein translocase. Nat Struct Mol Biol 2004; 11: 234-241.

3. Mokranjac D, Sichting M, Neupert W, Hell K. Tim14, a novel key component of the import motor of the TIM23 protein translocase of mitochondria. EMBO J 2003; 22: 4945-4956.

4. Schusdziarra C, Blamowska M, Azem A, Hell K. Methylation-controlled J-protein MCJ acts in the import of proteins into human mitochondria. Hum Mol Genet 2013; 22: 1348-1357.

5. Sinha D, Joshi N, Chittoor B, Samji P, D'Silva P. Role of Magmas in protein transport and human mitochondria biogenesis. Hum Mol Genet 2010; 19: 1248-1262.

6. Strathdee G, Davies BR, Vass JK, Siddiqui N, Brown R. Cell type-specific methylation of an intronic $\mathrm{CpG}$ island controls expression of the MCJ gene. Carcinogenesis 2004; 25: 693-701.

7. Lindsey JC, Lusher ME, Strathdee G, Brown R, Gilbertson RJ, Bailey S et al. Epigenetic inactivation of MCJ (DNAJD1) in malignant paediatric brain tumours. Int J Cancer 2006; 118: $346-352$.

8. Shridhar V, Bible KC, Staub J, Avula R, Lee YK, Kalli K et al. Loss of expression of a new member of the DNAJ protein family confers resistance to chemotherapeutic agents used in the treatment of ovarian cancer. Cancer Res 2001; 61: 4258-4265.

9. Gulbins E, Dreschers S, Bock J. Role of mitochondria in apoptosis. Exp Physiol 2003; 88: 85-90.

10. Fulda S, Galluzzi L, Kroemer G. Targeting mitochondria for cancer therapy. Nat Rev Drug Discov 2010; 9: 447-464.

11. Azzolin L, von Stockum S, Basso E, Petronilli V, Forte MA, Bernardi P. The mitochondrial permeability transition from yeast to mammals. FEBS Lett 2010; 584: 2504-2509.

12. Lemasters JJ, Theruvath TP, Zhong Z, Nieminen AL. Mitochondrial calcium and the permeability transition in cell death. Biochim Biophys Acta 2009; 1787: 1395-1401. 
13. Leung AW, Halestrap AP. Recent progress in elucidating the molecular mechanism of the mitochondrial permeability transition pore. Biochim Biophys Acta 2008; 1777: 946-952.

14. Meinecke M, Wagner R, Kovermann P, Guiard B, Mick DU, Hutu DP et al. Tim50 maintains the permeability barrier of the mitochondrial inner membrane. Science 2006; 312: 1523-1526

15. Rasola A, Sciacovelli M, Chiara F, Pantic B, Brusilow WS, Bernardi P. Activation of mitochondrial ERK protects cancer cells from death through inhibition of the permeability transition. Proc Natl Acad Sci USA 2010; 107: 726-731.

16. Giorgio V, von Stockum S, Antoniel M, Fabbro A, Fogolari F, Forte M et al. Dimers of mitochondrial ATP synthase form the permeability transition pore. Proc Natl Acad Sci USA 2013; 110: 5887-5892.

17. Zoratti M, Szabo I. The mitochondrial permeability transition. Biochim Biophys Acta 1995; 1241: $139-176$

18. Baines CP, Kaiser RA, Purcell NH, Blair NS, Osinska $\mathrm{H}$, Hambleton MA et al. Loss of cyclophilin $\mathrm{D}$ reveals a critical role for mitochondrial permeability transition in cell death. Nature 2005; 434: 658-662

19. Boettcher M, Kischkel F, Hoheisel JD. High-definition DNA methylation profiles from breas and ovarian carcinoma cell lines with differing doxorubicin resistance. PLoS One 2010; 5 e11002.

20. Dube H, Selwood D, Malouitre S, Capano M. Simone MI, Crompton M. A mitochondrialtargeted cyclosporin $\mathrm{A}$ with high binding affinity for cyclophilin $\mathrm{D}$ yields improved cytoprotection of cardiomyocytes. Biochem J 2012; 441: 901-907.

21. Petronilli V, Miotto G, Canton M, Colonna R, Bernardi P, Di Lisa F. Imaging the mitochondrial permeability transition pore in intact cells. Biofactors 1998; 8: 263-272.

22. Bernardi P, Scorrano L, Colonna R, Petronilli V, Di Lisa F. Mitochondria and cell death. Mechanistic aspects and methodological issues. Eur J Biochem 1999; 264: 687-701.

23. Huser J, Rechenmacher CE, Blatter LA. Imaging the permeability pore transition in single mitochondria. Biophys J 1998; 74: 2129-2137.

24. Berridge MJ, Lipp P, Bootman MD. The versatility and universality of calcium signalling Nat Rev Mol Cell Biol 2000; 1: 11-21.

25. Hajnoczky G, Csordas G, Das S, Garcia-Perez C, Saotome M, Sinha Roy S et al. Mitochondrial calcium signalling and cell death: approaches for assessing the role of mitochondrial Ca2 + uptake in apoptosis. Cell Calcium 2006; 40: 553-560.
26. Odagiri K, Katoh $\mathrm{H}$, Kawashima H, Tanaka T, Ohtani $\mathrm{H}$, Saotome M et al. Local control of mitochondrial membrane potential, permeability transition pore and reactive oxygen species by calcium and calmodulin in rat ventricular myocytes. J Mol Cell Cardiol 2009; 46 : 989-997.

27. Kang BH. TRAP1 regulation of mitochondrial life or death decision in cancer cells and mitochondria-targeted TRAP1 inhibitors. BMB Rep 2012; 45: 1-6.

28. Kang BH, Plescia J, Dohi T, Rosa J, Doxsey SJ, Altieri DC. Regulation of tumor cell mitochondrial homeostasis by an organelle-specific Hsp90 chaperone network. Cell 2007; 131: $257-270$.

29. Strathdee G, Vass JK, Oien KA, Siddiqui N, Curto-Garcia J, Brown R. Demethylation of the MCJ gene in stage III/IV epithelial ovarian cancer and response to chemotherapy. Gynecol Oncol 2005; 97: 898-903.

30. Jubinsky PT, Short MK, Mutema G, Morris RE, Ciraolo GM, Li M. Magmas expression in neoplastic human prostate. J Mol Histol 2005; 36: 69-75.

31. Salhab M, Patani N, Jiang W, Mokbel K. High TIMM17A expression is associated with adverse pathological and clinical outcomes in human breast cancer. Breast Cancer 2012; 19: $153-160$

32. Samudio I, Fiegl M, Andreeff M. Mitochondrial uncoupling and the Warburg effect: molecular basis for the reprogramming of cancer cell metabolism. Cancer Res 2009; 69: 2163-2166.

33. Kroemer G, Galluzzi L, Brenner C. Mitochondrial membrane permeabilization in cell death. Physiol Rev 2007; 87: 99-163.

34. Zhang C, Wu B, Beglopoulos V, Wines-Samuelson M, Zhang D, Dragatsis I et al. Presenilins are essential for regulating neurotransmitter release. Nature 2009; 460 : 632-636

(i) (2) Cell Death and Disease is an open-access journal published by Nature Publishing Group. This work is licensed under a Creative Commons Attribution-NonCommercialShareAlike 3.0 Unported License. To view a copy of this license, visit http://creativecommons.org/licenses/by-nc-sa/3.0/

Supplementary Information accompanies this paper on Cell Death and Disease website (http://www.nature.com/cddis) 